\title{
Celebrating anniversaries
}

\section{H. Emons}

Published online: 13 March 2014

(c) Springer-Verlag Berlin Heidelberg 2014

There are different habits in defining 'special anniversaries' Nowadays many organisations seem to follow a 5-year celebration cycle which demonstrates the rapidly changing conditions under which many of them are operating. Thus, reaching the 'silver anniversary', i.e. 25 years of existence, constitutes a real milestone, in particular for a voluntary organisation. Therefore, Accreditation and Quality Assurance (ACQUAL) would like to congratulate Eurachem, a network focusing on analytical chemistry in Europe, on this occasion. This provides also a special opportunity for some personal reflections on Eurachem and its activities.

Analytical chemistry has a long tradition and continues to be further developed and applied in university programs, non-academic research, industry, trade, societal services, regulatory activities etc. There are plenty of scientific organisations who intend to cover parts of analytical chemistry or even its whole bandwidth. One may wonder what is so special about a 'network of organisations providing a focus for analytical chemistry and quality-related issues in Europe', as stated on Eurachem's web page. For me, it is Eurachem's emphasis on the 'and' in that mission statement. The last 25 years have indeed seen a (qualitatively) new level in the combination of analytical chemistry and quality assurance. This can be noted at both the conception and the application stages. It is acknowledged that impressively accurate measurement results were paving the way for the scientific discipline 'analytical chemistry' from the very beginning and continue to push its limits. And there have been and will be laboratories which produce very reliable analytical information without running a

H. Emons ( $\square)$

Geel, Belgium

e-mail: JRC-IRMM-ACQUAL@ec.europa.eu formal quality management system. However, the more systematic and wider inclusion of appropriate quality assurance (QA) measures, based on metrological principles and best-practice experience, has allowed the expansion of analytical activities and the confidence building in results to a much larger scale. Documentary standards such as the former EN 45001, a precursor of ISO/IEC 17025, appeared in the 1990s and were used as benchmarks for a more harmonised third-party assessment of laboratories offering services in chemical analysis.

This rapid evolution of merging analytical chemistry with concepts and tools from general quality assurance, metrology, standardisation and accreditation has created significant demands for explanatory and guidance documents. Eurachem has identified such needs and has served the community of analytical chemists with a number of valuable guides. From my personal view and observation, the guides on method validation, quantifying uncertainty and traceability seem to be the most influential ones so far. I consider the efforts to mediate between the various technical languages used in analytical chemistry, metrology, quality management and auditing as a specific strength of those guides. This is not easy, and further promotion of common understanding is required, but Eurachem has established a reputation in this communication exercise.

Another merit of Eurachem consists for me in providing a platform for exchange and discussing issues regarding analytical quality assurance. Eurachem's workshops and working groups promote the spreading of good concepts and approaches. They are used to test ideas and build consensus. But they fulfil also an educational role and help to recruit analytical chemists into the QA community. Several of them have joined the group of Eurachem enthusiasts over the years. Their personal conviction, passion and commitment have shaped this voluntary network. 
It seems to be remarkable how different the standing of quality assurance in analytical chemistry is today in comparison with 25 years ago. Different drivers such as many new regulations and social concerns are demanding analytical results fit for decision-making. New national and international actors have appeared on the scene, just think about the growth and development of accreditation bodies. Multi- and interdisciplinary aspects and tasks form to a much larger extent than ever the daily business of analytical chemists, who have to defend their results towards the non-analysts (and often non-scientists). All these create, to my understanding, interesting but demanding challenges on quality in analytical chemistry. Several of the original goals of Eurachem may have yet not been sufficiently achieved, such as the systematic integration of analytical QA in academic teaching in Europe. Therefore, Eurachem continues to have an important role working towards these aims.

The following two papers in this issue of Accreditation and Quality Assurance have been written with the purpose of informing our readership about several aspects of the remarkable development of this 'network of organisations'. I take the opportunity to wish Eurachem all the best and continued support from many colleagues on its road to the 'golden anniversary'. This journal will continue providing a platform for its deliverables.

Hendrik Emons

Editor-in-Chief 